\title{
A Revised Received Signal Strength Based Localization for Healthcare
}

\author{
Wenhuan Chi ${ }^{1}$, Yuan Tian ${ }^{2}$, Mznah Al-Rodhaan ${ }^{2}$, Abdullah Al-Dhelaan ${ }^{2}$ and \\ Yuanfeng Jin ${ }^{1 *}$ \\ ${ }^{1}$ Department of Mathematics, Yanbian University, Yanji 133002, China \\ ${ }^{2}$ Department of Computer Science, King Saud University,Saudi \\ 843240086@qq.com,ytian@ksu.edu.sa,rodhaan@ksu.edu.sa, \\ dhelaan@ksu.edu.sa,yfkim@ybu.edu.cn,yfkim@ybu.edu.cn”
}

\begin{abstract}
Location-awareness is important for healthcare, and can be applied to the various consumer applications. The received signal strength (RSS) based localization technique has advantages of needing no additional hardware and simple to be implemented inbuilding applications. Received signal strength indication fingerprinting (RSSIFP) is an indoor localization technique. However, the RSS is affected by radio signals' reflections, shadowing, and fading. To solve this problem, an effective indoor localization method of revised RSSIFP is proposed to reduce the deviation during indoor RSSIFP localization. The proposed algorithm uses the RSSIFP based on the position probability grid. Before position, the RSSIFP data are revised according to anchor node signal and time tag. The $K$-nearest neighbor $(K N N)$ and weighted centre localization method is adopted in position prediction. A test-bed only including common consumer electronic equipments such as wireless access point (AP), Zigbee node and smart cell-phone is deployed. Performance results show that the proposed algorithm outperforms other algorithms in the healthcare environments.
\end{abstract}

Keywords: Localization, RSSI fingerprinting revised signal, KNN, Weighted centre localization, Healthcare

\section{Introduction}

Monitoring is the most important basic function for healthcare. Knowing the patient's location is useful for monitoring assistance. The localization in e-health environment is indoor localization, which is different from GPS based outdoor position. Wireless sensor network is an prominent tool to identify mobile node's positions in absolute or relative coordinates systems, which has been received increasing attention[1-3].

It's common of using wireless signal strength to estimate the locations of the moving devices based on the fixed reference devices with their location information. The classic methods to estimate the indoor location are time-of-arrival (TOA) [4], time-difference-ofarrival (TDOA) [5], angle-of-arrival (AOA) [6], and received signal strength (RSS) [7].

While the synchronization error will exist forever, TOA method will lead to exactly accurate because it is based on signal travel times between nodes. Avoiding this time error, TDOA method uses the difference of the signals' arrival time between anchor nodes, but an extra hardware (anchor nodes) is required. The angles between unknown node and a number of anchor nodes are used in the AOA method to estimate the location. TDOA, AOA also need extra hardware. However, the RSS based technique needs no additional hardware and it is simple to be implemented for in-building applications, and it has established the mathematical model on the basis of path loss attenuation with distance. RSS indication (RSSI) based localization is simple and the cost of implementation is

Corresponding Author 
much cheaper than TOA and AOA-based methods. It is suitable to be employed in the WSN environment.

Unfortunately, it is not easy for radio-based localization, because signal has reflection, diffraction and scattering characters. The studies showed radio waves' degrading effects of reflections, shadowing, and fading cause the large variability of RSS [8-11]. As a result, RSS based localization methods' accuracy is not guaranteed due to signals' errors and instability. However, there need no extra equipment attracts many researchers' eyes. In fact, most of smart mobile phones have a built-in RSSI, which provides RSS measurement without any extra cost.

Utilizing RSS method's simple, easy measure advantage and avoiding signal instable shortage, we will present a RSS methods combine few anchor nodes, which can lead to accurate location estimation in this paper. The anchor node is used to correct the errors caused by signal's instability. RSSI fingerprints are captured and k-nearest neighbor $(\mathrm{KNN})$ algorithm is adopted to find $\mathrm{k}$ locations that have a similar fingerprint. The accuracy of the proposed method is estimated in experiments.

\section{Related Works}

The majority of studies on RSS based localization have been performed [12]. However, the effect of spurious disturbances on the accuracy of RSS-based indoor localization is still existed. The RSS-based localization methods need no extra hardware, alternatively, they store coordinate information which is related to RSSI fingerprints. The RADAR [13] system illustrates the principle of RSS-based localization.

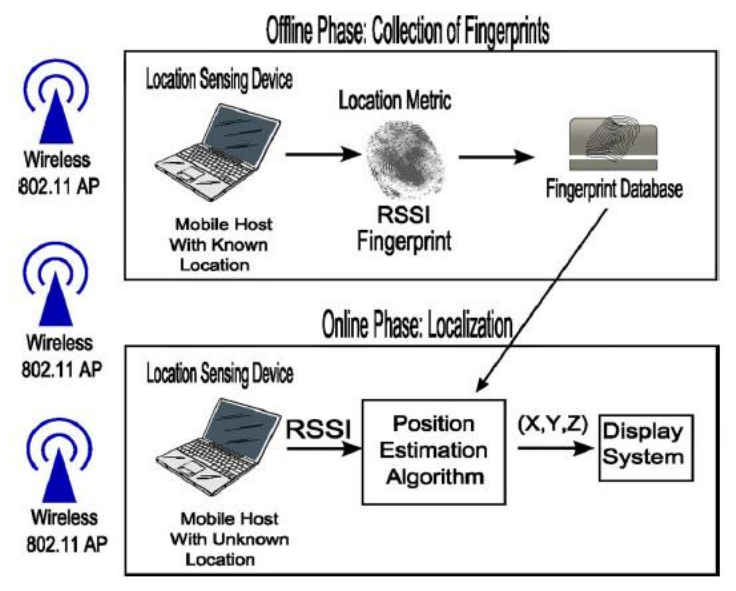

Figure 1. Overview of RSSI Fingerprinting

As shown in Figure 1, there are two phases. In the fingerprints capture phase, a map of RSSI fingerprints is constructed for a given floor. The floor has been divided into a grid of cells. For each cell, the fingerprint can be collected. In location prediction phase, portable device will read RSSI and use classification techniques to predict its location cell that has a similar fingerprint. Then, the predicted location will be shown on the map.

Based on RSS fingerprint, there exist several algorithms that can be used to determine the position of a target through RSS measurements. There are two types methods: geometric methods and statistical method. Minimum-maximum method (min-max) is the classic geometric method and the maximum likelihood method is the representative of statistical method.

Statistical method and artificial neural network method are proposed in [7] for distance measure. The experiments show that the most important factor for distance estimation is the transmission power according to the relevant distance. Min-Max algorithm is 
proposed to overcome the large attenuation measurement error for in-building wireless applications[2]. A parameter variations tolerance method is proposed in [14] and it is more accurate as time increases. A Sigma-Point Kalman Smoother (SPKS)-based location algorithm, which extend Kalman filter is proposed and it shows its superior in localization accuracy [15]. Hongliang and Meng [16] proposed a transmit-power adaptive localization algorithm based on particle filtering for sensor networks assisted by multiple transmitpowers.

As we know, RSSI value varies over time due to non-negligible multipath fading, especially in indoors environments. This will affect location measurement accuracy. As a perfect solution, different fingerprints in physical space have different signal values can decide position. Especially, the signal values are far apart which leads to good locate precision. For a system designer, it is most important to construct a map or fingerprint database for a given environment. As usually, an optimization fingerprint map will be decides by the number of AP (Access point), the location of AP, and the size of lattice. In this point, researchers have considered the use of strategically positioned anchors, theoretical modeling of signal space, and calibration to quickly build the database [17]. The RSSI database is build once a time, but the localization accuracy is different according to different algorithm. Brett and Chin [12] have surveyed several RSSIFP systems and divided localization algorithms into deterministic and probabilistic methods. In which, $k$-nearest neighbor and Bayesian algorithms represent deterministic and probabilistic methods accordingly. Also, found AP number affected the localization accuracy from $2.2 \mathrm{~m} 38 \%$ with three APs [13] to 3m 90\% with 33APs [17]. Future more, Barsocchi et al [18] found that APs tend to drift in and out of range frequently, and proved only a subset of APs is used for localization.

\section{Research Methodology}

As we know, the RSS is influenced by reflections, shadowing, and fading of radio waves. The indoor environment is complex and in which the wave is a Non-Line-Sight transfer. In the same position, the RSS is dynamically changeable according to different time, temperature, humidity, and movement objects etc. So, traditional RSSI fingerprints used to location prediction results errors. The stability of receiving signal strength is essential for position predication. The general flow of revised signal strength localization method is: RSSI fingerprint collection, RSSI revising, position prediction.

\section{A. Infrastructure of Test-Bed}

Figure 2 depicts the floor plan of experimental site in ubiquitous life care research center, where the floor size is $6 \mathrm{~m} \times 9 \mathrm{~m}$. The system infrastructure was composed of one mobile node (i.e., the target), one fixed node (i.e., anchor node), four APs in the corner of room. The floor was divided into 260 test points located on a grid with a resolution of 50 $\mathrm{cm}$. The fixed node was hanged on the center of ceiling which is $260-\mathrm{cm}$ high, which is represented with squares in Figure 2.

There is a home gateway server receiving the localization request and the RSS change message. In practice, the mobile node sends a message to home gateway when its received RSSI is changed. The home gateway requests anchor node to send RSSI information when it receives message from mobile node. The home gate way receives RSSI measurement from mobile node and anchor node, and then, it makes mobile location prediction based on stored RSSI fingerprint and deterministic algorithm. 


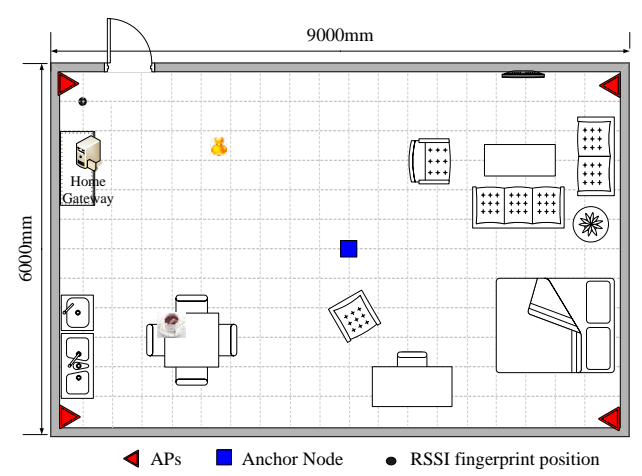

(a)

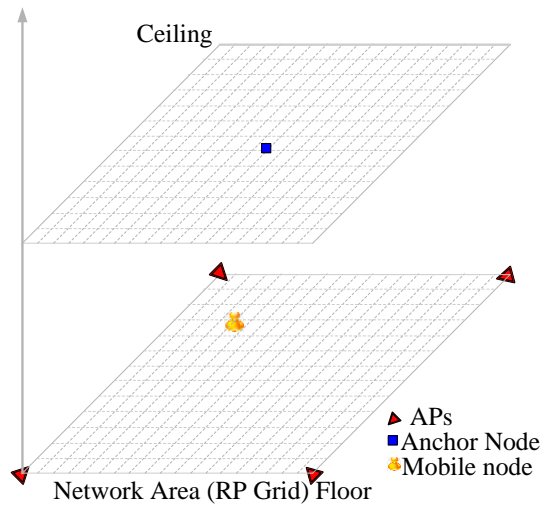

(b)

Figure 2. Healthcare Test-Bed (a) System Deployment, (b) Fingerprint Position Grid Cell

\section{B. Data Collection}

In order to minimize the exchange of messages and the data processing time, data collection should be performed on the anchor node and mobile node, while data processing should be executed in home gateway as a personal computer allows an exhaustive statistical analysis.

In this phase, the RSSI fingerprint data are collected for 260 test points. In order to identify all available APs, the 802.11 spectrum all channels are scanned. Also, the time varying effect should be removed and a robust picture of the APs' signal strength are built. We use WiFi application programming interface (API) to capture the RSSI values in $\mathrm{dBm}$.

The measuring process was repeated 100 times for each of the 260 test point locations, resulting in a total amount of $104000 \mathrm{RSS}$ values collected. The achieved measurement repeatability was quite high, i.e., always within $\pm 1 \mathrm{dBm}$. The average value of each AP is used to represent as RSSI fingerprint. For the $260 \mathrm{RP}$ (Reference Point) points, the RSSI information was grasped every $1 \mathrm{~s}$ over a period of $2 \mathrm{~min}$. After each $2 \mathrm{~min}$ scanning, the average RSSI for each AP for a certain RP position is inserted into the fingerprint database. A truncated example of this file is shown in Table 1.

Table 1. Fingerprint RSSI Values (in dBm)

\begin{tabular}{c|cccccc}
\hline Num & $R P$ & $A P 1$ & $A P 2$ & $A P 1$ & $A P 2$ & time \\
\hline $\mathbf{1}$ & $(0,0)$ & -52 & -31 & -73 & -60 & $2013-01-2609: 56$ \\
$\mathbf{2}$ & $(0,1)$ & -49 & -29 & -70 & -57 & $2013-01-2609: 56$ \\
$\mathbf{3}$ & $(1,0)$ & -40 & -25 & -66 & -53 & $2013-01-2609: 56$ \\
$\ldots$ & $\ldots$ & $\ldots$ & $\ldots$ & $\ldots$ & $\ldots$ & $\ldots$ \\
$\mathbf{2 6 0}$ & $(12,19)$ & -69 & -75 & -31 & -49 & $2013-01-2609: 56$ \\
\hline
\end{tabular}

\section{Fingerprint Correction}

The signal is fluctuant even though the indoor environment is unchanged. The signal strength is normal distributed without any interference. So, in fingerprint database, we use mean value over 100 times as the RSS value to overcome the signal's instability. But for the localization, we need measure the RSS and make prediction immediately; average value is not acceptable for real time requirement. Even though the environment is unchanged, the RP's RSSI change with the signal power from AP time to time. So, we need construct a map function from real time signal power to RSSI fingerprint data.

While in fingerprint measurement, we capture the anchor node's fingerprint $A_{0}$ and RP's fingerprint $F P$ in a mean value. We note it as $A_{0}=\left[a_{1}, a_{2}, \cdots, a_{n}\right]$, while $a_{i}$ is the mean RSSI value from the $i$-th AP, $n$ is the number of AP. The RSSI fingerprint data is noted 
as:

$$
F P=\left[\begin{array}{cccc}
f p_{1,1} & f p_{1,2} & \cdots & f p_{1, n} \\
f p_{2,1} & f p_{2,2} & \cdots & f p_{2, n} \\
\vdots & \vdots & \vdots & \vdots \\
f p_{m, 1} & f p_{m, 2} & \cdots & f p_{m, n}
\end{array}\right]
$$

Where $m$ is the $R P$ point number.

While the mobile node sends out localization requirement, the mobile node and the anchor node get the RSSI values as $M=\left[m_{1}, m_{2}, \cdots, m_{n}\right]$ and $A^{\prime}=\left[a_{1}^{\prime}, a_{2}^{\prime}, \cdots, a_{n}^{\prime}\right]$ accordingly. We use the newly received RSSI $A^{\prime}$ of the anchor node compared with $A_{0}$ to correct the fingerprint FP data FP.

$$
f p_{i, j}=\frac{a_{j}}{a_{j}} f p_{i, j}
$$

Where $i=1, \cdots m, j=1, \cdots n$

Through this step, the mean RSSI based fingerprint database FP is transferred into a time based fingerprint database $F P^{\prime}$. In this system, the corrected fingerprint data are stored as a multi-copy with time tag. As a result, there are many fingerprint copies in the system. As the signal will change over time, the localization can make precise prediction with different fingerprints according to time stamp.

\section{Position Prediction}

The localization problem based on fingerprint can shortly be formalized as follows. Consider the set of nodes $\mathrm{N}=\left\{\mathrm{RP}_{1}, \mathrm{RP}_{2}, \ldots, \mathrm{RP}_{\mathrm{n}}\right\}$, i.e., each one with a fixed and known position (hence the name reference position). The reference position is described in 2-D localization since the third dimension usually is not of primary interest in an indoor environment. Thus, the position of a reference is $R P_{i}=\left(x_{i}, y_{i}\right)$, where $x_{i}$ and $y_{i}$ are evaluated with respect to origin $O$. Let $p$ denote the position of a mobile node of unknown coordinates $x, y$. The goal of an RSS based localization algorithm is to provide estimate $p=(x, y)$ of position $p$ given the vector $M=\left[m_{1}, m_{2}, \cdots, m_{n}\right]$.

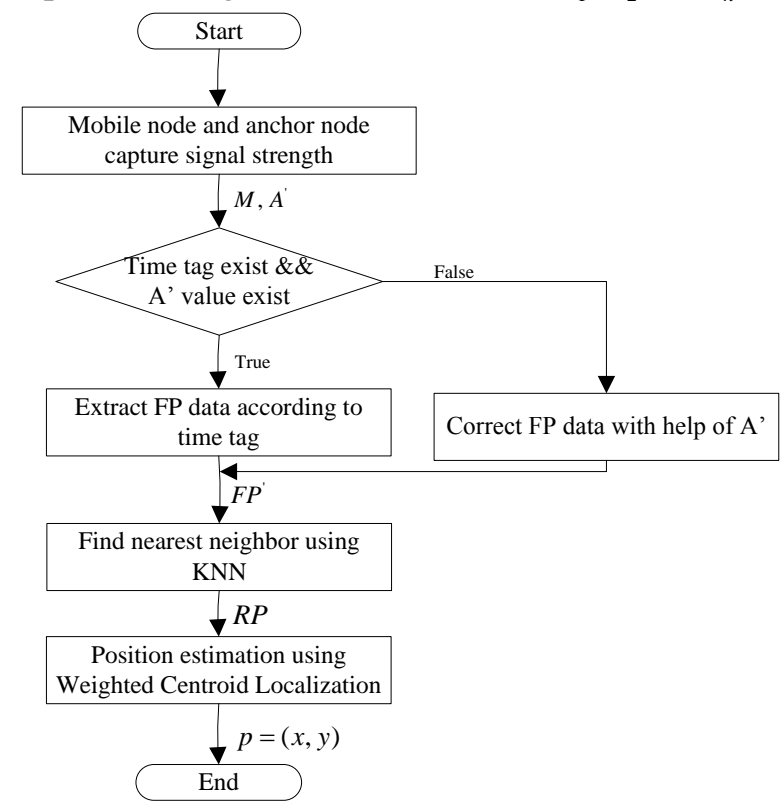

Figure 3. The Flow Chart of Localization 
We can divide the position prediction into two steps: the KNN (k-nearest neighbor) reference nodes selection, and the position estimation.

(1) KNN based RP Selection

The reference node set is gotten based on the RSSI distance between mobile node's $M=\left[m_{1}, m_{2}, \cdots, m_{n}\right]$ and any RP point's $F P_{i}=\left[f p_{i, 1}, f p_{i, 2} \cdots, f p_{i, m}\right]$.

$$
\text { Dis }=\sqrt{\sum_{j=1}^{n}\left(f p_{i, j}-m_{j}\right)^{2}}
$$

Also, we know the strong signal strength is useful to localization but the weak signal will cause more deviation. It means different signal strength has different contribution for position prediction. The weight of each RSS is calculated as:

$$
w_{j}=\frac{\left|m_{n-j}\right|}{\left|\sum_{j=1}^{n} m_{j}\right|}
$$

While the RSSI is a negative value, the weaker strength the RSSI is smaller, but the absolute value is bigger. So, using $\left|m_{n-j}\right|$ represents the importance of $m_{j}$.

So, the weighted signal strength distance is:

$$
\text { Dis }=\sqrt{\sum_{j=1}^{n} \frac{\left|m_{n-j}\right|}{\left|\sum_{j=1}^{n} m_{j}\right|}\left(f p_{i, j}-m_{j}\right)^{2}}
$$

The KNN algorithm is used to calculate the signal distances, which between mobile node and RP points, and choose the smallest four distances RP points as the neighbor points.

(2) Position Estimation

Position estimation is calculating the mobile node $p(x, y)$ based on the 4 nearest neighbor RP nodes mentioned above. We adopt weighted centroid localization (WCL) [19] algorithm to calculate the centroid of the coordinates $R P_{i}=\left(x_{i}, y_{i}\right)$ of the so-called KNN $\mathrm{RP}$ position, which are stored in fingerprint database. More specifically, the estimated position of the mobile node is given by:

$$
p=\frac{1}{k} \sum_{i=1}^{k} R P_{i}
$$

Where $k$ is the number of the nearest neighbor RP position of mobile node. It is worth noticing that the centroid results the center of the $R P_{i}$ coordinates. It means that the $p$ is the center of its four nearest points.

Actually, equation (5) is most likely to be unsatisfied in practice because it assumes all the neighbor nodes are equally near the mobile node, in [19], the introduction of a function, which assigns a greater weight to the points closest to the mobile node, was proposed. The result is the WCL algorithm, which estimates the position of the target node as:

$$
p=\sum_{i=1}^{k}\left(R P_{i} * \frac{D i s_{k-i}}{\sum_{i=1}^{k} D i s_{i}}\right)
$$

\section{Experimental Results}

We implemented the proposed localization algorithm based on the Wifi. In our Lab, there are four wireless access point which are deployed to send out signal and one Zigbee based node hung in the ceiling to receive the signal. The user is equipped with smart cell 
phone which acts as mobile node. The server based home gateway will receive the requests, process the data and make position prediction.

The wireless access points are deployed at corners as shown in Figure 2, two on desk about 1 meter above floor, two on the floor. As there is some furnitures in the home, the signal sent from floor has larger attenuation as shown Figure 4. This experiment was executed at a fixed point to measure the signals from four APs about 60 minutes. Thus, the signal of AP1 and AP2 are more stable than AP3, AP4.

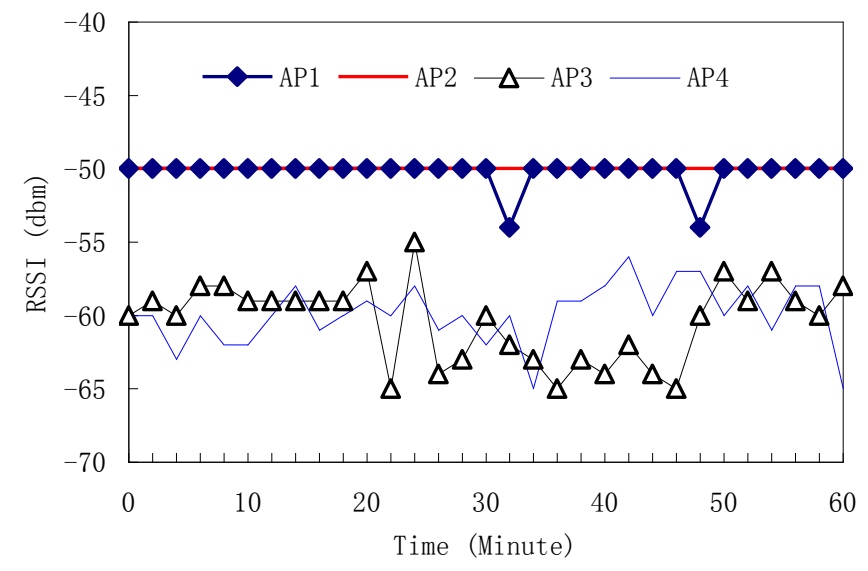

Figure 4. RSSI Measurements in the Mobile Device

From the Figure 4, we know the signal of AP1, AP2 are suitable for localization. However, this is a static environment. For healthcare, the elders will move and need making prediction. So, the moving object is not considered means the human body is not considered. In the next simulation, we not only use these two APs, but we also consider the other two APs which are affected by attenuation largely.

We make a comparison between the traditional fingerprint (FP) localization algorithm, signal revised fingerprint ( $\mathrm{rFP}$ ) localization algorithm, and WCL based signal revised fingerprint (wrFP) localization algorithm. For measurement of the accuracy of the estimated position for each localization algorithm, the root mean square distance error (RMSE) is used.

$$
R M S E=\sqrt{\frac{1}{n} \sum_{i=1}^{n}\left[\left(\hat{x}_{i}-x_{i}\right)^{2}+\left(\hat{y_{i}}-y_{i}\right)^{2}\right]}
$$

Where $n$ is the number of position the mobile node visited, the $\left(x_{i}, y_{i}\right)$ and $\left(x_{i}, y_{i}\right)$ are the real and the estimated position of the mobile node position $i$. During the experiment, 30 positions $(n=30)$ are chosen randomly to verify the algorithm's effects. The results are shown in table 2 and Figure 5.

Table 2. Localization Error According to different Number of AP

\begin{tabular}{|c|c|c|c|c|c|c|}
\hline \multirow{2}{*}{$\begin{array}{c}\text { Number } \\
\text { of AP }\end{array}$} & \multicolumn{2}{|c|}{$F P$} & \multicolumn{2}{|c|}{$r F P$} & \multicolumn{2}{|c|}{$w r F P$} \\
\hline & Max error & Mean error & Max error & Mean error & Max error & Mean error \\
\hline 2 & 2.86 & 2.35 & 1.40 & 1.32 & 1.49 & 1.23 \\
\hline 3 & 2.76 & 1.83 & 1.58 & 1.08 & 1.30 & 0.90 \\
\hline 4 & 2.40 & 2.12 & 1.63 & 1.20 & 1.40 & 1.00 \\
\hline
\end{tabular}

Table 2 shows the max position error and mean error for each algorithm according to different AP which has been adopted

From the results, the wrFP algorithm adopting three AP is the best choice for our 
experiment.

Also, table 2 indicates that the localization precision is about 1 meter even though our gird solution is 0.5 meter. This is because our AP is deployed indoors and our experiment environment is not wide enough. In addition, the RSSI values between FP points are not apart enough. The no distinguished RSSI values will cause the localization error. Compared to literature [12], our results is better.

Theoretically, the more AP will generate high localization precision. However, for our environment, the three AP is better than Four AP, because the AP3 and AP4 fluctuate greatly. The more fluctuant signal will decrease the prediction accuracy.

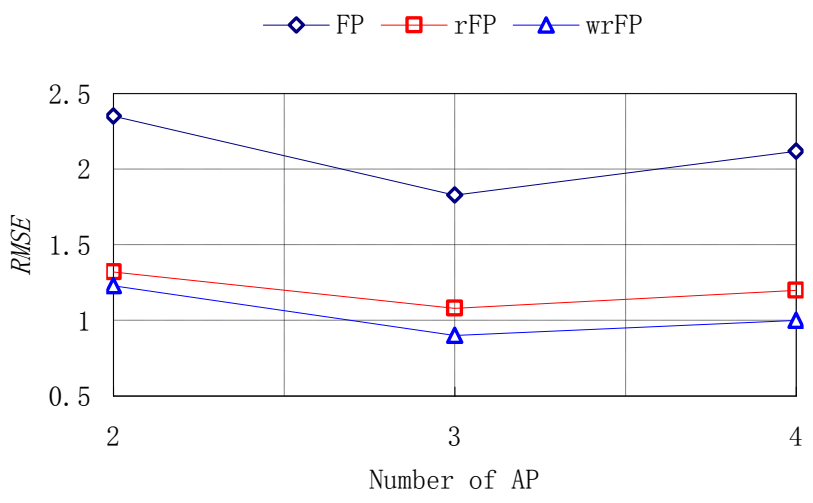

\section{Figure 5. Localization Error of Comparing Various Algorithms According to} different Number of AP

Figure 5 indicates that the revised signal based fingerprint algorithm decreases the localization error sharply, compared to traditional fingerprint algorithm. The wrPF algorithm presented here has the least localization error which is less than 1 meter. In most cases, wrPF algorithm localization accuracy is much closed to rPF whose localization error is about 1 to 1.5 meters.

\section{Conclusion}

The new signal revised RSSI based localization algorithm is proposed in this paper. The basic approach is to divide the network area into the small grids and perform the localization using the position grid. Based on traditional RSSIFP method, an anchor node is adopted as a RSS correction reference for the fingerprint database. This anchor node can remove the signal attenuation in a better way. We construct a test-bed using simple equipments and get a good performance. The experiments show that the proposed approach can achieve high location estimation accuracy for in-building wireless localization applications.

\section{Acknowledgements}

This work was supported in part by China Postdoctoral Science Foundation (No. 2012M511783), National Science Foundation of China (No. 61173143, 11361066), and Special Public Sector Research Program of China (No. GYHY201206030) and was also supported by PAPD.

The authors extend their appreciation to the Deanship of Scientific Research at King Saud University for funding this work through research group no. RGP-264. 


\section{References}

[1] C. Long, W. C. Dong and Z. Y. Zhou, "Indoor robot localization based on wireless sensor network", Consumer Electronics, IEEE Transactions on, vol. 57, (2011), pp. 1099-1104.

[2] K. Youngbae, K. Younggoo and P. GwiTae, "Robust localization over obstructed interferences for inbuilding wireless applications", Consumer Electronics, IEEE Transactions on, vol. 55, (2009), pp. 105-111.

[3] C. Y. Yi and L. Y. Yuan, "A new received signal strength based location estimation scheme for wireless sensor network", Consumer Electronics, IEEE Transactions on, vol. 55, (2009), pp. 1295-1299.

[4] W. Yunbo, S. Goddard and L. C. Perez, "A study on the cricket location-support system communication protocols", in Electro/Information Technology, 2007 IEEE International Conference on, (2007), pp. 257-262.

[5] C. Blandin, A. Ozerov and E. Vincent, "Multi-source TDOA estimation in reverberant audio using angular spectra and clustering", Signal Processing, vol. 92, no. 8, pp. (2012), pp. 1950-1960.

[6] P. Kułakowski, J. V. Alonso, E. E. López, W. Ludwin and J. G. Haro, "Angle-of-arrival localization based on antenna arrays for wireless sensor networks", Computers \& Electrical Engineering, vol. 36, no. 11, (2010), pp. 1181-1186.

[7] A. Awad, T. Frunzke and F. Dressler, "Adaptive Distance Estimation and Localization in WSN using RSSI Measures", in Digital System Design Architectures, Methods and Tools, 2007. DSD 2007. 10th Euromicro Conference on, (2007), pp. 471-478.

[8] E. Elnahrawy, L. Xiaoyan and R. P. Martin, "The limits of localization using signal strength: a comparative study", in Sensor and Ad Hoc Communications and Networks, 2004. IEEE SECON 2004. 2004 First Annual IEEE Communications Society Conference on, (2004), pp. 406-414.

[9] K. Langendoen and N. Reijers, "Distributed localization in wireless sensor networks: a quantitative comparison," Computer Networks, vol. 43, pp. 499-518, 11/15/ 2003.

[10] D. Lymberopoulos, Q. Lindsey, and A. Savvides, "An Empirical Characterization of Radio Signal Strength Variability in 3-D IEEE 802.15.4 Networks Using Monopole Antennas," in Wireless Sensor Networks. vol. 3868, K. Römer, H. Karl, and F. Mattern, Eds., ed: Springer Berlin Heidelberg, 2006, pp. 326-341.

[11] N. Patwari, J. N. Ash, S. Kyperountas, A. O. Hero, R. L. Moses and N. S. Correal, "Locating the nodes: cooperative localization in wireless sensor networks", Signal Processing Magazine, IEEE, vol. 22, (2005), pp. 54-69.

[12] B. Dawes and K.-W. Chin, "A comparison of deterministic and probabilistic methods for indoor localization," Journal of Systems and Software, vol. 84, no. 3, (2011), pp. 442-451.

[13] P. Bahl and V. N. Padmanabhan, "RADAR: an in-building RF-based user location and tracking system", in INFOCOM 2000. Nineteenth Annual Joint Conference of the IEEE Computer and Communications Societies. Proceedings. IEEE, vol.2, (2000), pp. 775-784.

[14] A. H. Sung and Y. Wonpil, "Environmental-Adaptive RSSI-Based Indoor Localization," Automation Science and Engineering", IEEE Transactions on, vol. 6, (2009), pp. 626-633.

[15] A. S. Paul and E. A. Wan, "RSSI-Based Indoor Localization and Tracking Using Sigma-Point Kalman Smoothers", Selected Topics in Signal Processing, IEEE Journal of, vol. 3, (2009), pp. 860-873.

[16] R. Hongliang and M. Q. H. Meng, "Power Adaptive Localization Algorithm for Wireless Sensor Networks Using Particle Filter", Vehicular Technology, IEEE Transactions on, vol. 58, (2009), pp. 2498-2508.

[17] O. M. Badawy and M. A. B. Hasan, "Decision Tree Approach to Estimate User Location in WLAN Based on Location Fingerprinting", in Radio Science Conference, 2007. NRSC 2007. National, (2007), pp. 1-10.

[18] P. Barsocchi, S. Lenzi, S. Chessa and G. Giunta, "Virtual Calibration for RSSI-Based Indoor Localization with IEEE 802.15.4", in Communications, 2009. ICC '09. IEEE International Conference on, (2009), pp. 1-5.

[19] J. Blumenthal, R. Grossmann, F. Golatowski and D. Timmermann, "Weighted Centroid Localization in Zigbee-based Sensor Networks", in Intelligent Signal Processing, 2007. WISP 2007. IEEE International Symposium on, (2007), pp. 1-6. 


\section{Author}

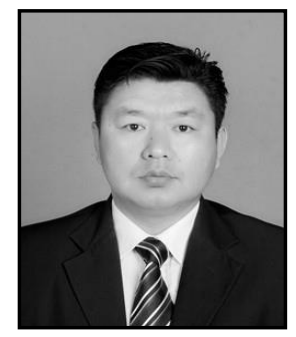

Yuanfeng Jin, Associate Professor Yanbian University, China, Main activities and responsibilities Lecture on numerical computation \& PDE Research on parallel computation. 